\title{
OPTIMAL MULTIPLE STOPPING MODELS OF RELOAD OPTIONS AND SHOUT OPTIONS
}

\author{
MIN DAI* AND YUE KUEN KWOK ${ }^{\dagger}$
}

\begin{abstract}
The reload provision in an employee stock option entitles its holder to receive one new (reload) option from the employer for each share tendered as payment of strike upon the exercise of the stock option. The number of reloads allowed can be finite or infinite. The shout feature in a call option allows its holder to reset the option's strike price to the prevailing stock price upon shouting. We explore symmetry relationship between the price functions of the employee reload options and shout call options. When the dividend yield of the underlying stock is zero, the value of the employee reload option can be expressed in terms of the price functions of a shout call option and forward contract. For an employee reload option with an infinite number of allowable reloads, the payoff of the employee reload option can be related to the lookback feature of the stock price process. We also examine the optimal exercise policies of the multi-reload employee stock options. The behaviors of the critical stock price at which the holder should exercise optimally are shown to depend on the relative magnitude of the dividend yield, interest rate and volatility. Our analysis of multi-reload and multi-shout options contributes to the literature on optimal stopping policies of contingent claims with multiple stopping rights.
\end{abstract}

Key words. Employee Stock Options, Reload Feature, Shout Call Options, Multiple Optimal Stopping, Lookback Options

AMS subject classifications. Mathematics Subject Classifications (1991): 90A09, 91B28, 93E20

1. Introduction. With the specific aim to provide better incentive compensation for the employees, employee stock options are structured to include non-typical provisions that are not commonly found in conventional exchange-traded or over-thecounter options. Johnson and Tain [11] examine the incentive effects of five different types of non-traditional executive stock option plans, including the performance vested stock options, repriceable (reset) stock options, reload stock options and others. In this paper, we concentrate on the theoretical analysis of the optimal stopping policies of the reload provision in employee stock options. Our studies reveal the interesting linkage between three types of exotic option features, namely, reload, shout (strike reset) and lookback features. Under some special cases, we show that the price functions of reload options are related to those of call options with shout (strike reset) feature and floating strike lookback options.

The reload provision allows the employee stock option holder to exercise an inthe-money stock option prior to expiration. The strike is paid using owned shares of the employee. To compensate for the potential loss of future price appreciation on the shares tendered for strike payment, the employee is granted with the same number of new (reload) options as the number of shares tendered. The strike price of the reload option is then reset at the prevailing stock price at the exercise moment. In this sense, the employee can lock in the gain on the part that is in-the-money at the exercise moment while continues retaining the potential upside growth of the stock price through holding the reload options. For example, suppose the strike price of the original option is $\$ 100$, and the prevailing stock price at the exercise moment is $\$ 150$.

\footnotetext{
*Department of Mathematics, National Singapore University, Singapore 117543 (matdm@nus. edu.sg).

${ }^{\dagger}$ Correspondence author. Department of Mathematics, Hong Kong University of Science and Technology, Clear Water Bay, Hong Kong, China (maykwok@ust.hk).
} 
This means $\$ 100 / \$ 150=2 / 3$ units of owned share would be tendered to pay as strike upon exercise. Under the reload provision, the holder will be granted $2 / 3$ units of new option, with the same expiration date as the original option and the strike price set at $\$ 150$.

In real practice, more contractual complexities can be incorporated into the reload provision. The discussion of various types of reload option plans can be found in the paper by Hemmer et al. [9]. The number of reloads throughout the life of the stock option can be finite or infinite. Under the time vesting clause, the reload provision cannot be activated during the early period of the stock options. In addition, after each reload, the next reload can be activated only after a vesting period has lapsed. Also, one may include the performance vesting clause whereby the reload provision is triggered only when the stock price has breached certain threshold value from below. Actual examples of firms issuing employee reload can be readily obtained by a search in the National Automated Accounting Search System.

The additive incentive effects of the reload feature in employee stock options appear to be uncertain and more in-depth theoretical and empirical studies are required for better understanding. It is generally speculated that the reload feature may lower the dead weight loss, which is the difference between the cost of granting the option and the employee's private value. Also, reload programs are believed to be useful at increasing company ownership among participants.

The apparent complex structures in the reload provision appear to cause its valuation infeasible. In particular, the employee's private value depends on his own risk aversion level. This has led the Financial Accounting Standards Board [7] to conclude that it is not feasible to estimate the value of an option with the reload feature when the right can be exercised at any time. According to the study made by the management compensation consultant company, Frederick W. Cook \& Co., "Reloads appear destined to continue their fate as one of the most misunderstood compensation tools, utilized by a slowly increasing minority of companies". The true test of the reload effectiveness is its survivability, that is, whether reload programs continue to survive after companies have tried them out. So far, the answer seems to be quite positive, according to the survey done by Frederick W. Cook \& Co. [8].

Several papers have appeared in the literature which consider the numerical algorithms for valuation of employee stock options under the Black-Scholes paradigm. To price the value of the optimal exercise right embedded in an option using the binomial scheme, it is well known that one may adopt the dynamic programming procedure of comparing the continuation value and exercise value in each binomial node. Similar dynamic programming procedure have been applied to evaluate the optimal exercise policies and calculate the value of employee stock options with reload rights (Hemmer et al., [9]; Saly et al., [14]). The employee reload option becomes a compound option if the right to reload occurs only once on a preset date within the life of the option. Under such restriction, it becomes straightforward to derive the price formula for the reload option (Johnson and Tian, [11]).

The paper by Dybvig and Loewenstein [6] contributes to the major breakthrough on the understanding of the pricing behaviors of employee reload options. They consider reload options with infinite reload rights and the employee can choose to exercise at any time during the life of the reload option. Under these assumptions, they show that the value of the reload option always lies between the value of an American call and the stock price, even with infinite reloads and perpetual life of the option. Also, the total number of new shares issued under all exercises is always 
bounded by the initial number of options issued. With infinite reloads allowed, they prove that the optimal policy is to reload whenever a new maximum value of the stock price is realized. Accordingly, the value of the reload option is given by the risk neutral expectation of a payoff function that depends on the maximum of the original strike and the realized maxima of the stock price over the life of the option. In addition, they discuss the impact of the time vesting requirement on the price function and optimal exercise policy of the reload option.

In this paper, we consider the valuation of the market value of the employee reload option and adopt the Black-Scholes pricing framework, which implicitly assumes zero transaction costs and that the stock options can be traded and hedged. This may be somewhat not reflecting the full nature of employee stock options, where employees are constrained not to short sell the company stocks and the options are non-transferable. The market value does provide an upper bound to the value of the option to the holder. We take advantage of the nice analytic tractability of the BlackScholes pricing framework so as to reveal some of the pricing behaviors of the reload feature. When we consider the private value of the reload option to the employee, it is necessary to include the risk aversion level of the employee into the pricing framework. For example, an employee who is risk averse would diversify his wealth from strong dependence on the company fortune by exercising the option at a lower stock price level. The pricing approach under the utility maximization framework has been considered by Lau and Kwok [12].

We consider employee reload options where the number of reloads can be finite or infinite, and the reload can be exercised at any time during the life of the option. Our analysis of optimal exercise policies of multi-reload options contributes to the recent literature on valuation of contingent claims with multiple stopping rights $[1,2$, $10,13]$. The pricing model in this paper does not include the time and performance vesting requirements. The analytic tractability will be much reduced when vesting requirements are included, and only numerical solution of the pricing model is feasible. The construction of the corresponding numerical algorithm for pricing reload option with time vesting is considered in our other paper (Dai and Kwok, [5]).

A shout call option refers to a call option with the embedded right that the holder can choose to shout at any moment during the life of the option to install a lower strike price, which is set to be the prevailing stock price at the shouting moment (Thomas, [15]; Dai et al. [4]). The maximum number of resets allowed in a shout call can be finite or infinite. It is obvious that the holder shouts only at stock price level that is below the strike price set at the earlier shout. If there has been no shout commencing throughout the whole life, then the strike price remains at the original value set at initiation. Without vesting requirement, we illustrate that there exists symmetry relations between the reload provision and the right held by the holder to reset the strike price. When the dividend yield of the underlying stock is zero, we show that the value of the reload option can be expressed in terms of the price functions of a forward contract and a shout call option.

When the employee stock option contains the provision of infinite reloads, the corresponding option model can be shown to be related to a floating strike lookback options. Similar relation is seen for shout options with infinite reset rights (Dai et al. [4]). These results are obtained by virtue of the linear complementarity formulation of option pricing model with optimal exercise right. Dybvig and Loewenstein [6] obtain the valuation formulas of infinite-reload options by assuming reloads to be allowed at distinct time instants, then taking the number of allowable instants to be 
infinite. They use dominance argument to derive the optimal exercise policy, which maximizes both the market value as well as the value of holder's private valuation. In our work, we start with pricing models for options with finite reloads and take the limit of number of reloads to infinity to obtain the solution for infinite-reload options. We also examine the characterization of the optimal reload policies for the employee options with finite and infinite reloads. The critical stock price at which it is optimal to exercise the reload right can exhibit different characteristics, depending on the relative magnitude of the interest rate, dividend yield and volatility. The asymptotic value of the critical stock price at infinite time to expiry may be finite or infinite. Under certain set of parameter values in the pricing model, it may be never optimal to exercise when the time to expiry exceeds certain threshold value.

The paper is organized as follows. In Section 2, we present the linear complementarity formulation of employee stock options and shout call options with finite number of reloads/shouts allowed. When the dividend yield of the stock is zero, we derive the price formula of the reload option in terms of the price function of a shout call option. The properties of the critical stock price at which the holder should exercise the reload provision are analyzed. We also explore the monotonicity properties of the price functions and critical stock prices with respect to the number of reloads. In Section 3, we analyze the employee stock options with infinite number of reloads, and in particular, we establish their linkage with the lookback options. We also derive explicit valuation formulas for the infinite-reload option under different dividend policies of the underlying stock. In Section 4, we present our numerical calculations that were performed to verify the theoretical results obtained in Sections 2 and 3. The paper is ended with conclusive remarks in the last section.

2. Optimal stopping policies with finite reloads and shouts. In this section, we consider the pricing models of employee reload options and shout call options with finite number of reloads or shouts. Let $S$ denote the stock price and assume that the stock pays dividend at the constant yield $q$. Let $r$ denote the risk free interest rate and $t$ be the current time. We adopt the usual Black-Scholes pricing framework. Under the risk neutral valuation framework, the stock price process is assumed to follow the lognormal process

$$
\frac{d S}{S}=(r-q) d t+\sigma d Z
$$

where $\sigma$ is the constant volatility and $Z$ is the standard Wiener process. We concentrate our analysis on the employee reload options and examine how the pricing models of the reload options are related to those of the shout call options. Let $T$ denote the expiration date of the employee option and $X$ denote the original strike price, and write $\tau=T-t$ as the time to expiry. We assume that the option received in the last reload is European style. Let $V_{n}(S, \tau ; X, r, q)$ denote the price function of the employee reload option with $n$ reloads allowed throughout the life of the option. In Sec. 2.1, we determine the price function of the reload option with single reload and explore in details the corresponding optimal exercise policy. We then generalize the pricing formulation of reload options with $n$ reloads in Sec. 2.2. We examine the monotonicity properties of the price functions and the critical stock price $S_{n}^{*}(\tau)$ at which it is optimal to exercise. Also, we explore the impact of dividend yield $q$ on $V_{n}(S, \tau ; X, r, q)$ and $S_{n}^{*}(\tau)$.

2.1. Single-reload and single-shout call options. Upon the exercise of the reload right, the employee receives one unit of stock and pays the strike price $X$. It is 
assumed that the employee uses $\frac{X}{S}$ units of owned stock for the strike payment so that the number of units of new stock received upon exercise is essentially $1-\frac{X}{S}$. Further, we assume that the employee keeps these new stock so that continuous dividend yields will be received. In addition, the employee receives $\frac{X}{S}$ units of new call option with strike price set at the prevailing stock price at the exercise moment and same maturity date $T$. With the single reload right utilized, there will be no more reload allowed so that the new option is just the usual European call option. Let $c(S, \tau ; X, r, q)$ denote the price function of a European call. Upon exercise, the value of the reload option becomes $S-X+\frac{X}{S} c(S, \tau ; S, r, q)$. By virtue of the Black-Scholes price formula of European call option and observing the linear homogeneity property of the call price function with respect to $S$, we obtain

$$
c(S, \tau ; S, r, q)=S \widehat{c}(\tau ; r, q),
$$

where

$$
\widehat{c}(\tau ; r, q)=e^{-q \tau} N\left(\widehat{d}_{1}\right)-e^{-r \tau} N\left(\widehat{d}_{2}\right) .
$$

Here, $N(x)$ denotes the cumulative normal distributive function defined by

$$
N(x)=\frac{1}{\sqrt{2 \pi}} \int_{-\infty}^{x} e^{-t^{2} / 2} d t
$$

and

$$
\widehat{d}_{1}=\frac{r-q+\frac{\sigma^{2}}{2}}{\sigma} \sqrt{\tau} \quad \text { and } \quad \widehat{d}_{2}=\frac{r-q-\frac{\sigma^{2}}{2}}{\sigma} \sqrt{\tau} .
$$

Let $p(S, \tau ; X, r, q)$ denote the price function of a European put option. In a similar manner, we have

$$
p(S, \tau ; S, r, q)=S \widehat{p}(\tau ; r, q)
$$

where

$$
\widehat{p}(\tau ; r, q)=e^{-r \tau} N\left(-\widehat{d}_{2}\right)-e^{-q \tau} N\left(-\widehat{d}_{1}\right) .
$$

\section{Linear complementarity formulation}

The pricing model of options with reload/shout right can be formulated in terms of the linear complementarity formulation. We present the corresponding formulation for (i) single-reload employee option, (ii) single-shout call option.

\section{Single-reload option}

The payoff function upon exercise of the single-reload option is $S-X+X \widehat{c}(\tau ; r, q)$ [see Eq. (2.2)], with the presence of the extra term $X \widehat{c}(\tau ; r, q)$ in the payoff when compared with the exercise payoff of an American call. The linear complementarity formulation for the price function $V_{1}(S, \tau ; X, r, q)$ takes the form (Wilmott et al., [16])

$$
\begin{aligned}
& \frac{\partial V_{1}}{\partial \tau}-\mathcal{L}_{r, q} V_{1} \geq 0, \\
& V_{1}(S, \tau) \geq S-X+X \widehat{c}(\tau), \\
& {\left[\frac{\partial V_{1}}{\partial \tau}-\mathcal{L}_{r, q} V_{1}\right]\left\{V_{1}(S, \tau)-[S-X+X \widehat{c}(\tau)]\right\}=0,} \\
& \qquad S \in(0, \infty), \tau \in(0, T],
\end{aligned}
$$


where the differential operator $\mathcal{L}_{r, q}$ is given by

$$
\mathcal{L}_{r, q}=\frac{\sigma^{2}}{2} S^{2} \frac{\partial^{2}}{\partial S^{2}}+(r-q) S \frac{\partial}{\partial S}-r, \quad r>0 \quad \text { and } \quad q \geq 0 .
$$

The terminal payoff condition is given by $V_{1}(S, 0)=(S-X)^{+}$, where

$$
x^{+}=\left\{\begin{array}{ll}
x & \text { if } x \geq 0 \\
0 & \text { if } x<0
\end{array} .\right.
$$

The domain of the pricing model $\{(S, \tau): 0 \leq S \leq \infty, 0 \leq \tau \leq T\}$ is divided into the continuation region $\mathcal{C}$ and the stopping region $\mathcal{S}$, which are separated by the critical boundary $S_{1}^{*}(\tau), 0 \leq \tau \leq T$. The employee should optimally choose to exercise the reload right when the stock price is equal to or greater than $S_{1}^{*}(\tau)$. In the stopping region, $V_{1}=S-X+X \widehat{c}(\tau ; r, q)$; and accordingly,

$$
\begin{aligned}
& \left(\frac{\partial}{\partial \tau}-\mathcal{L}_{r, q}\right)[S-X+X \widehat{c}(\tau ; r, q)] \\
= & q S-r X+X \frac{d \widehat{c}}{d \tau}(\tau ; r, q)+r X \widehat{c}(\tau ; r, q) .
\end{aligned}
$$

Single-shout call option

Let $c_{\text {shout }, 1}(S, \tau ; X, r, q)$ denote the price function of a single-shout call option. Upon shouting, the strike price $X$ is reset to the prevailing stock price so that the payoff upon shouting becomes a European call option whose price function is given by $c(S, \tau ; S, r, q)$. By virtue of the linear homogeneity property, the payoff can be simplified to become $S \widehat{c}(\tau ; r, q)$. The linear complementarity formulation for $c_{\text {shout }, 1}$ $(S, \tau ; X, r, q)$ is given by

$$
\begin{aligned}
& \frac{\partial c_{\text {shout }, 1}}{\partial \tau}-\mathcal{L}_{r, q} c_{\text {shout }, 1} \geq 0 \\
& c_{\text {shout }, 1}(S, \tau) \geq S \widehat{c}(\tau ; r, q), \\
& {\left[\frac{\partial c_{\text {shout }, 1}}{\partial \tau}-\mathcal{L}_{r, q} c_{\text {shout }, 1}\right]\left[c_{\text {shout }, 1}(S, \tau)-S \widehat{c}(\tau ; r, q)\right]=0,} \\
& S \in(0, \infty), \quad \tau \in(0, T] .
\end{aligned}
$$

The terminal payoff condition is given by $c_{\text {shout }, 1}(S, 0)=(S-X)^{+}$, like that of the usual call option. Let $\widehat{S}_{1}^{*}(\tau ; r, q)$ denote the critical stock price of the single-shout call. The holder of the single-shout call should shout optimally whenever $S \leq \widehat{S}_{1}^{*}(\tau ; r, q)$.

Next, we will show that the price functions of the single-reload option and singleshout call option are closely related when $q=0$. With the presence of dividend yields, unfortunately, no simple relation between the price functions can be established.

\section{Zero dividend yield}

When the stock pays zero dividend yield, we show that the price function of a singlereload option can be expressed in terms of the price functions of a forward contract and the single-shout call option. Since the exercise payoff of the single-reload option contains the term $S-X$, we attempt to simplify the pricing formulation through the subtraction of the price function of a forward contract from $V_{1}(S, \tau ; X, r, 0)$. By defining

$$
U_{1}(S, \tau ; X, r, 0)=V_{1}(S, \tau ; X, r, 0)-\left(S-X e^{-r \tau}\right)
$$


and observing the following relation that arises from the put-call parity

$$
X \widehat{c}(\tau ; r, 0)-\left(X-X e^{-r \tau}\right)=X \widehat{p}(\tau ; r, 0),
$$

one can show that $U_{1}(S, \tau ; X, r, 0)$ is governed by

$$
\begin{aligned}
& \frac{\partial U_{1}}{\partial \tau}-\mathcal{L}_{r, 0} U_{1} \geq 0, \\
& U_{1}(S, \tau) \geq X \widehat{p}(\tau ; r, 0), \\
& {\left[\frac{\partial U_{1}}{\partial \tau}-\mathcal{L}_{r, 0} U_{1}\right]\left[U_{1}(S, \tau)-X \widehat{p}(\tau ; r, 0)\right]=0,} \\
& \quad S \in(0, \infty), \tau \in(0, T], \\
& U_{1}(S, 0)=(X-S)^{+} .
\end{aligned}
$$

Suppose we define

$$
x=\frac{X}{S} \quad \text { and } \quad W_{1}(x, \tau)=\frac{1}{S} U_{1}(S, \tau) .
$$

By observing the put-call symmetry relation:

$$
\widehat{p}(\tau ; r, 0)=\widehat{c}(\tau ; 0, r),
$$

then $W_{1}(x, \tau)$ satisfies the following linear complementarity formulation

$$
\begin{aligned}
& \frac{\partial W_{1}}{\partial \tau}-\mathcal{L}_{0, r} W_{1} \geq 0 \\
& W_{1}(x, \tau) \geq x \widehat{c}(\tau ; 0, r), \\
& {\left[\frac{\partial W_{1}}{\partial \tau}-\mathcal{L}_{0, r} W_{1}\right]\left[W_{1}(x, \tau)-x \widehat{c}(\tau ; 0, r)\right]=0,} \\
& \quad x \in(0, \infty), \tau \in(0, T], \\
& W_{1}(x, 0)=(x-1)^{+} .
\end{aligned}
$$

The payoff upon exercise is $x \widehat{c}(\tau ; 0, r)=c(x, \tau ; x, 0, r)$, which is a European call option with strike price set at the prevailing stock price. By comparing Eq. (2.14) with Eq. (2.9), we observe that $W_{1}(x, \tau)$ is governed by the same linear complementarity formulation that is satisfied by the price function of the one-shout call option with zero interest rate, unit strike price, dividend yield $r$. In summary, we obtain

$$
V_{1}(S, \tau ; X, r, 0)=S-X e^{-r \tau}+c_{\text {shout }, 1}(X, \tau ; S, 0, r),
$$

where $c_{\text {shout }, 1}(S, \tau ; X, r, q)$ is the price function of the one-shout call option.

The shout call option is a simpler product compared to the reload option. By virtue of the above decomposition, analysis of the optimal exercise policy for the reload option becomes easier by simply analyzing the optimal exercise policy for the shout call counterpart.

Properties of the critical stock price $S_{1}^{*}(\tau ; r, q)$

Similar to holding an American call option, the employee chooses to exercise optimally the reload option when the stock price rises above some critical stock price $S_{1}^{*}(\tau)$. The stopping region and the continuation region correspond to $S \geq S_{1}^{*}(\tau)$ and $S<S_{1}^{*}(\tau)$, 
respectively. The characterization of the optimal exercise policy of the single-reload option is summarized in Theorem 1.

\section{Theorem 1}

Let $S_{1}^{*}(\tau ; r, q)$ denote the critical stock price of single-reload employee stock option that separates the stopping and continuation regions. The stopping region and $S_{1}^{*}(\tau)$ observe the following properties.

1. The stopping region is contained inside the region

$$
\{(S, \tau): S \geq X, \quad 0 \leq \tau \leq T\} .
$$

2. At time close to expiry,

$$
S_{1}^{*}\left(0^{+} ; r, q\right)=X, \quad q \geq 0, r>0 .
$$

3. When the stock pays dividend at constant yield $q>0$, the critical stock price at infinite time to expiry is given by

$$
S_{1}^{*}(\infty ; r, q)=\frac{\mu_{+}}{\mu_{+}-1} X
$$

where $\mu_{+}$is the positive root of the equation:

$$
\frac{\sigma^{2}}{2} \mu^{2}+\left(r-q-\frac{\sigma^{2}}{2}\right) \mu-r=0
$$

4. If the stock pays no dividend, then

(a) for $r \leq \frac{\sigma^{2}}{2}, S_{1}^{*}(\tau ; r, 0)$ is defined for all $\tau>0$ and $S_{1}^{*}(\infty ; r, 0)=\infty$;

(b) for $r>\frac{\sigma^{2}}{2}, S_{1}^{*}(\tau ; r, 0)$ is defined only for $0<\tau<\tau_{1}^{*}$, where $\tau_{1}^{*}$ is the unique solution to the algebraic equation

$$
-r N\left(-\frac{r+\frac{\sigma^{2}}{2}}{\sigma} \sqrt{\tau}\right)+\frac{\sigma}{2 \sqrt{\tau}} n\left(-\frac{r+\frac{\sigma^{2}}{2}}{\sigma} \sqrt{\tau}\right)=0
$$

where

$$
n(x)=\frac{1}{\sqrt{2 \pi}} e^{-x^{2} / 2} .
$$

The proof of Theorem 1 is presented in Appendix A. As a remark, unlike the reset put option analyzed by Dai et al. [4], the behaviors of the critical stock price of the single-reload option depend on the relative magnitude of $r$ and $\sigma^{2} / 2$ instead of $r$ and $q$.

2.2. Multi-reload options. Most of the results established earlier for singlereload options can be extended to multi-reload options. When the stock pays no dividend, the price function of a $n$-reload option can be expressed as the sum of the price functions of the corresponding forward contract and $n$-shout call option. Intuitively, one would expect that the holder will exercise their reload right at a lower critical stock price when there are more reload rights outstanding. In general, it would be difficult to obtain explicit valuation formulas for the price functions when the number of reloads is more than one. Without explicit formulas, the proof of 
monotonicity properties on the critical stock prices would be infeasible. We resort to numerical calculations to verify the optimal exercise policies and monotonicity properties on the critical stock prices for multi-reload options (see Section 3). For $q>0$, one can derive the recursive relation that relates the critical stock prices for perpetual multi-reload options [see Eq. (2.21)].

The multi-reload option is a nice example of contingent claims with multiple stopping rights. Another class of contingent claims with multiple stopping are the swing options in energy derivatives. Theoretical aspects of optimal stopping policies of swing options are discussed by Carmona and Touzi [1,2] while methods of numerical valuation of multiple-exercise contingent claims are presented by Ibanez [10] and Meinshausen and Hambly [13]. The complementarity formulation for $V_{n}(S, \tau ; X, r, q)$ takes similar form as that shown in Eq. (2.6)

$$
\begin{aligned}
& \frac{\partial V_{n}}{\partial \tau}-\mathcal{L}_{r, q} V_{n} \geq 0 \\
& \quad V_{n}(S, \tau ; X) \geq S-X+X V_{n-1}(1, \tau ; 1), \\
& \left(\frac{\partial V_{n}}{\partial \tau}-\mathcal{L}_{r, q} V_{n}\right)\left\{V_{n}(S, \tau ; X)-\left[S-X+X V_{n-1}(1, \tau ; 1)\right]\right\}=0, \\
& \quad S \in(0, \infty), \tau \in(0, T], \\
& V_{n}(S, 0)=(S-X)^{+} .
\end{aligned}
$$

The above pricing formulation is valid for $n \geq 1$, and for notational convenience, we assume $V_{0}(S, \tau ; X, r, q)$ to be $c(S, \tau ; X, r, q)$. Note that $V_{n}(1, \tau ; 1)$ is a time dependent function that is obtained by setting $S=1$ and $X=1$ in the price function $V_{n}(S, \tau ; X)$. As the option with more reload rights outstanding should be more expensive than its counterpart with less reload rights, we expect that $V_{n+1}(S, \tau ; X)>V_{n}(S, \tau ; X)$ for all $S>0$ and $\tau>0$.

When the stock does not pay dividend, a simple relation between the price functions of the $n$-reload option and $n$-shout call option can be found. We apply the transformation

$$
W_{n}(x, \tau)=\frac{1}{S}\left[V_{n}(S, \tau ; X, r, 0)-\left(S-X e^{-r \tau}\right)\right] \quad \text { and } \quad x=\frac{X}{S}
$$

to obtain

$$
\begin{aligned}
& \frac{\partial W_{n}}{\partial \tau}-\mathcal{L}_{0, r} W_{n} \geq 0 \\
& W_{n}(x, \tau) \geq x W_{n-1}(1, \tau), \\
& {\left[\frac{\partial W_{n}}{\partial \tau}-\mathcal{L}_{0, r} W_{n}\right]\left[W_{n}(x, \tau)-x W_{n-1}(1, \tau)\right]=0,} \\
& \quad x \in(0, \infty), \quad \tau \in(0, T] \\
& W_{n}(x, 0)=(x-1)^{+} .
\end{aligned}
$$

Like the single-reload option and single-shout call option [see Eq. (2.15)], we can establish similar symmetry relation

$$
V_{n}(S, \tau ; X, r, 0)=S-X e^{-r \tau}+c_{\text {shout }, n}(X, \tau ; S, 0, r),
$$

where $c_{\text {shout }, n}(S, \tau ; X, r, q)$ is the price function of a $n$-shout call option. 
Properties of the critical stock price $S_{n}^{*}(\tau ; r, q)$

The critical price $S_{n}^{*}(\tau ; r, q)$ of a $n$-reload employee stock option exhibits similar behaviors as those of the single-reload option. The holder should never exercise at $S<X$ and $S_{n}^{*}(\tau ; r, q)$ starts at $X$ as $\tau \rightarrow 0^{+}$. Also, we observe the monotonic property: $S_{n+1}^{*}(\tau ; r, q)<S_{n}^{*}(\tau ; r, q)$, an obvious fact from financial intuition (see Dai et al.'s paper [3] on the discussion of the monotonic properties of the price functions of options with multiple reset rights). When $q=0$, the optimal exercise policy of a $n$-reload option can be related directly to that of the $n$-shout call counterpart. When $q>0$, we obtain the following recursive relation on the critical stock price of the perpetual $n$-reload option:

$$
\frac{S_{n}^{*}(\infty)}{X}=\frac{\mu_{+}}{\mu_{+}-1}-\frac{1}{\mu_{+}-1}\left[\frac{S_{n-1}^{*}(\infty)}{X}\right]^{1-\mu_{+}}, \quad n>1 .
$$

Further, it can be shown that $S_{n}^{*}(\infty)$ is monotonically decreasing with respect to $n$ and $\lim _{n \rightarrow \infty} S_{n}^{*}(\infty)=X$. For the proof of these properties on $S_{n}^{*}(\infty)$, see Appendix B.

Since $S_{\infty}^{*}(\tau)$ is a non-decreasing function of $\tau$ and $S_{\infty}^{*}(\tau) \geq X$ for $\tau \geq 0$, and together with $S_{\infty}^{*}(\infty)=X$, we deduce that $S_{\infty}^{*}(\tau)=X$ for $\tau \geq 0$. This is a striking result. The optimal exercise policy of an infinite-reload option then becomes deterministic. Hence, the pricing of an infinite-reload option is no longer a free boundary value problem.

3. Employee stock options with infinite reloads and lookback options. In this section, we derive the analytic price formula for an infinite-reload option for $q \geq 0$. The success of the analytic tractability of the infinite-reload option as compared to its finite-reload counterparts stems from the simple exercise policy of the infinitereload option - exercise whenever the option is in-the-money. Such optimal exercise policy has been proven by Dybvig and Loewenstein [6] through the construction of a portfolio that replicates the infinite-reload option. They derive the price formulas for the infinite-reload options by assuming that exercise is available only on a set of preset times, then subsequently deduce the solution for continuous exercise of reloads by taking the limit of infinite number of exercisable times. By taking the number of reloads $n$ to be infinite in the linear complementarity formulation, we show that the resulting pricing formulation becomes closely related to that of a lookback option model.

We represent the price function of the infinite-reload option by $V_{\infty}(S, \tau ; X, r, q)$, which is seen to be $\lim _{n \rightarrow \infty} V_{n}(S, \tau ; X, r, q)$. Consider the function

$$
F(S, \tau)=S e^{-q \tau}-X e^{-r \tau}+q \int_{0}^{\tau}\left(S e^{-q u}-X e^{-r u}\right) d u,
$$

which satisfies the equation

$$
\begin{aligned}
& \frac{\partial F}{\partial \tau}-\mathcal{L}_{r, q} F=q(S-X) \\
& F(S, 0)=S-X .
\end{aligned}
$$

Note that $F(S, \tau)$ reduces to the price function of a forward contract when $q=0$. We define the transformation

$$
U_{\infty}(S, \tau ; X, r, q)=V_{\infty}(S, \tau ; X, r, q)-F(S, \tau),
$$


then $U_{\infty}(S, \tau ; X, r, q)$ is seen to satisfy the following linear complementarity formulation

$$
\begin{aligned}
& \frac{\partial U_{\infty}}{\partial \tau}-\mathcal{L}_{r, q} U_{\infty} \geq q(X-S) \\
& U_{\infty}(S, \tau ; X) \geq X U_{\infty}(1, \tau ; 1) \\
& {\left[\frac{\partial U_{\infty}}{\partial \tau}-\mathcal{L}_{r, q} U_{\infty}-q(X-S)\right]\left[U_{\infty}(S, \tau ; X)-X U_{\infty}(1, \tau ; 1)\right]=0} \\
& \quad S \in(0, \infty), \quad \tau \in(0, T] \\
& U_{\infty}(S, 0)=(X-S)^{+}
\end{aligned}
$$

Furthermore, we define

$$
W_{\infty}(x, \tau)=\frac{1}{S} U_{\infty}(S, \tau) \quad \text { and } \quad x=\frac{X}{S} .
$$

The corresponding pricing formulation for $W_{\infty}(x, \tau)$ can be expressed as

$$
\begin{aligned}
& \frac{\partial W_{\infty}}{\partial \tau}-\mathcal{L}_{q, r} W_{\infty} \geq q(x-1) \\
& W_{\infty}(x, \tau) \geq x W_{\infty}(1, \tau) \\
& {\left[\frac{\partial W_{\infty}}{\partial \tau}-\mathcal{L}_{q, r} W_{\infty}-q(x-1)\right]\left[W_{\infty}(x, \tau)-x W_{\infty}(1, \tau)\right]=0} \\
& \quad x \in(0, \infty), \quad \tau \in(0, T] \\
& W_{\infty}(x, 0)=(x-1)^{+} .
\end{aligned}
$$

Let $c_{\text {float }}(S, m, \tau ; r, q)$ denote the price function of a floating strike lookback call option with terminal payoff $S-m$, where $m$ is the realized minimum value of the stock price over the life of the option. Interestingly, $W_{\infty}(x, \tau)$ is related to $c_{f l o a t}(S, m, \tau ; r, q)$ through the following relation:

$$
\begin{aligned}
S W_{\infty}(x, \tau) & =c_{\text {float }}(X, \min (S, X), \tau ; q, r) \\
& +q \int_{0}^{\tau} c_{\text {float }}(X, \min (S, X), u ; q, r) d u
\end{aligned}
$$

the proof of which is presented in Appendix C.

Collecting all the above relations together, we obtain the price formula for an infinite-reload option with dividend yield $q$ as follows:

$$
\begin{aligned}
& V_{\infty}(S, \tau ; X, r, q) \\
= & S W_{\infty}\left(\frac{X}{S}, \tau\right)+F(S, \tau) \\
= & c_{f l o a t}(X, \min (S, X), \tau ; q, r)+\left(S e^{-q \tau}-X e^{-r \tau}\right) \\
& +q \int_{0}^{\tau}\left[c_{\text {float }}(X, \min (S, X), u ; q, r)+\left(S e^{-q u}-X e^{-r u}\right)\right] d u .
\end{aligned}
$$

In Appendix D, we show the equivalence of this pricing formula with that given by Dybvig and Loewenstein [6]. In conclusion, the price function of the infinite-reload option can be expressed in terms of the price functions of a forward and a floating strike lookback call option. 
4. Numerical calculations. We performed numerical calculations to check the validity of the results discussed in the last two sections. Also, we explore monotonicity properties on the price functions of multi-reload and infinite-reload options. The numerical results on the critical stock prices and price functions are obtained using the binomial scheme coupled with the dynamic programming procedure to incorporate the reload feature. At each binomial node, we take the maximum among the continuation value and the exercise value (see similar numerical procedures reported by Hemmer et al. [9] and Saly et al. [14]).

\section{Critical stock prices}

We calculated the critical stock price $S_{n}^{*}(\tau ; r, q), n=1,2,3$, under the following three cases (i) $q=0, r>\sigma^{2} / 2$, (ii) $q=0, r \leq \sigma^{2} / 2$, and (iii) $q>0$. In Figure 1 , we plot $S_{n}^{*}(\tau ; r, q)$ against $\tau$ for options with one, two and three reloads. The parameter values used in the calculations are: $q=0, r=0.1, \sigma=0.3$ and $X=1$. When the stock does not pay dividend and $r>\sigma^{2} / 2$, there exists a threshold value $\tau_{n}^{*}$ such that the holder never exercise when the time to expiry exceeds that value. Given the same time to expiry, options with more reload rights should be exercised at a lower critical stock price. Hence, we would expect the monotonicity property: $\tau_{1}^{*}<$ $\tau_{2}^{*}<\tau_{3}^{*}$. From the plots in Figure 1, these threshold values of time to expiry are found to be $\tau_{1}^{*}=6.78, \tau_{2}^{*}=12.38$ and $\tau_{3}^{*}=17.86$. In Figure 2 , we show the plot of $S_{n}^{*}(\tau ; r, q), n=1,2,3$, against $\tau$ corresponding to $q=0$ and $r \leq \sigma^{2} / 2$. The parameter values used in the calculations are: $q=0, r=0.04, \sigma=0.3$ and $X=1$. Apparently, these plots agree with the result that $S_{n}^{*}(\tau ; r, q)$ tends to infinity at infinite time to expiry. Also, we observe the monotonicity property: $S_{1}^{*}(\tau)<S_{2}^{*}(\tau)<S_{3}^{*}(\tau)$ for all $\tau>0$. When the stock pays dividend, the critical stock price is defined for all $\tau>0$, and according to Eq. (2.21), $S_{n}^{*}(\infty)$ is finite. Based on the set of parameter values: $q=0.03, r=0.04, \sigma=0.3$ and $X=1$, we obtained $S_{1}^{*}(\infty)=3.45, S_{2}^{*}(\infty)=1.99$ and $S_{3}^{*}(\infty)=1.59$, using the recursive relation (2.21). The curves of $S_{n}^{*}(\tau), n=1,2,3$, against $\tau$ in Figure 3 reveal the validity of these theoretical results. In all the plots of the critical stock price in the above three figures, we observe that $S_{n}^{*}(\tau)$ always starts at $X$ at $\tau \rightarrow 0^{+}$and increases monotonically with respect to $\tau$.

\section{Monotonicity properties of the price functions}

We also performed numerical calculations on the dependence of the price functions of employee stock options on stock price volatility. The plots of the option value against volatility are shown in Figure 4 . The set of parameter values used in the calculations are: $q=0.03, r=0.04, \tau=10$ and $S=X=1$. The price functions are monotonically increasing with respect to the number of reloads outstanding and volatility. Our calculations reveal that the infinite-reload option can be $20-25 \%$ more expensive than its one-reload counterpart.

5. Conclusion. In this paper, we derive some interesting symmetry relations between the three exotic option feature: reload, shout and lookback. Our theoretical studies contribute to a broader understanding of the pricing behaviors under the Black-Scholes paradigm of the employee stock options with finite or infinite number of reloads. The analysis of optimal policies of multiple stopping of contingent claims is mathematically challenging. We unravel the linkage between the reload provision in an employee stock option and the strike reset right in a shout call option. By establishing symmetry relation between the price functions of reload option and shout call option, we show that the optimal stopping policies of the reload options are identical to those of the shout call options. 
For employee options with finite reloads, we give detailed characterization of the optimal exercise policies and examine the monotonicity properties of the price functions and critical stock prices with respect to the number of reloads. When the stock pays no dividend, it is interesting to observe that the optimal stopping policy depends on the relative magnitude of the riskless rate and volatility. When the square of volatility is less than twice the value of riskless interest rate, the holder should never exercise at any stock price level when the time to expiry is above certain threshold level. Such behaviors of optimal stopping of the reload options distinguish from those of the reset put options, whose optimal stopping policies have dependence on the relative magnitude of the dividend yield and riskless interest rate only. When the dividend yield is non-zero, we obtain explicit formulas for the critical stock price of perpetual multi-reload options. We also illustrate through numerical experiment that reload options with more reload rights outstanding should be worth more and the holder should exercise the reload right at a lower critical stock price. The infinitereload employee options and lookback options are seen to be closely related. We provide valuation formulas for infinite-reload options with or without dividend yield of the underlying stock.

The relation between the infinite-reload (infinite-shout) right and lookback feature exhibits interesting mathematical characteristics. With finite reloads, the pricing model is one-dimensional but non-linear due to the presence of the free boundary as exemplifed by the non-deterministic critical stock price. However, when we take the limit of infinite reloads, the pricing model becomes linear since the critical stock price always equals the original strike price. However, the dimension of the model increases by one due to the inclusion of the extra lookback variable in the model formulation.

\section{REFERENCES}

[1] R. CARMONA AND N. TOUZI, Optimal multiple stopping and valuation of swing options, to appear in Mathematical Finance (2006).

[2] R. CARMONA AND S. DAYANIK, Optimal multiple-stopping of linear diffusions and swing options, Woring paper of Princeton University, (2003).

[3] M. DAI, Y.K. KWOK AND L. WU, Option with multiple reset rights, International Journal of Theoretical and Applied Finance, 6(6) (2003), pp. 637-653.

[4] M. DAI, Y.K. KWOK AND L. WU, Optimal shouting policies of options with strike reset right, Mathematical Finance, 14(3) (2004), pp. 383-401.

[5] M. DAI AND Y.K. KWOK, Valuing employee reload options under time vesting requirement, Quantitative Finance, 5(1) (2005), pp. 61-69.

[6] P.H. DYBVIG And M. LOEWENSTEIN, Employee reload options: Pricing, hedging and optimal exercise, Review of Financial Studies, 16(1) (2003), pp. 145-171.

[7] FINANCIAL ACCOUNTING STANDARDS BOARD. Accounting for stock-based compensation. Statement of Financial Accounting Standards no. 123 (1995).

[8] FREDERIC W. COOK AND COMPANY, Reload stock options - the first ten years. Working paper, Frederic W. Cook and Company (1998).

[9] T. HEMMER, S. MATSUNAGE AND T. SHEVLIN, Optimal exercise and the cost of granting employee stock options with a relaod provision, Journal of Accounting Research, 36, (1998) pp. 231-255.

[10] P.A. IBÁÑEZ, Valuation of simulation of contingent claims with multiple early exericse opportunities, Mathematical Finance, 14(2) (2004), pp. 223-248.

[11] S.A. JOHNSON AND Y.S. TIAN, The value and incentive effects of nontraditional executive stock option plans, Journal of Finance Economics, 57 (2000), pp. 3-34.

[12] K.W. LAU AND Y.K. KWOK, Valuation of empolyee reload options under utility maximization framework. To appear in International Journal of Theoretical and Applied Finance.

[13] N. MEINSHAUSEN AND B.M. HAMBLY, Monte Carlo methods for the valuation of multipleexercise options, Mathematical Finance, 14(4), (2004) pp. 557-583. 
[14] P.J. SALY, R. JAGANNATHAN, AND S.J. HUDDART, Valuing the reload features of executive stock options, Accounting Horizons, 13, (1999) pp. 219-240.

[15] B. THOMAS, Something to shout about, Risk, 6(May) (1993), pp. 56-58.

[16] P. WILMOTT, J.N. DEWYNNE and S.D. HOWISON, Option pricing: Mathematical Models and Computation, Oxford Financial Press, (1993). 


\section{APPENDIX A - Proof of Theorem 1}

1. When the single-reload option exercises its reload right, it reduces to its European call counterpart. To show that it is never optimal to exercise when $S<X$, it suffices to prove that when $S<X$, the exercise payoff is always less than the value of the European call option [which in turn less than $\left.V_{1}(S, \tau ; X, r, q)\right]$. Consider the difference

$$
\begin{aligned}
D(S, \tau)= & c(S, \tau ; X, r, q) \\
& -[S-X+X \widehat{c}(\tau ; r, q)],
\end{aligned}
$$

we observe that

$$
\frac{\partial}{\partial S} D(S, \tau)=N\left(d_{1}\right)-1<0
$$

and

$$
\left.D(S, \tau)\right|_{S=X}=0
$$

where

$$
d_{1}=\frac{\ln \frac{S}{X}+\left(r-q+\frac{\sigma^{2}}{2}\right) \tau}{\sigma \sqrt{\tau}} .
$$

This gives $D(S, \tau)>0$ for $S<X$. Hence, the stopping region is contained in $\{(S, \tau): S \geq X$ and $0 \leq \tau<T\}$.

2. According to the linear complementarity formulation in Eq. (6), the difference

$$
\widehat{D}(S, \tau)=V_{1}(S, \tau ; X, r, q)-[S-X+X \widehat{c}(\tau ; r, q)]
$$

should be always positive for all $\tau>0$ in the continuation region. We prove the result: $S^{*}\left(0^{+} ; r, q\right)=X$ by contradiction. Suppose $S^{*}\left(0^{+}\right)>X$ and for $S \in\left(X, S^{*}\left(0^{+}\right)\right)$, we observe $\widehat{D}(S, 0)=0$ and

$$
\frac{\partial \widehat{D}}{\partial \tau}\left(S, 0^{+}\right)=-X\left[\frac{d \widehat{c}}{d \tau}\left(0^{+} ; r, q\right)+r \widehat{c}\left(0^{+} ; r, q\right)\right]<0 .
$$

This leads to $\widehat{D}(S, \tau)<0$, for $\tau$ close to $0^{+}$. A contradiction is encountered, so we should have $S^{*}\left(0^{+}\right) \leq X$. Together with the result: $S_{1}^{*}(\tau) \geq X$ proved in part (1), we conclude that $S^{*}\left(0^{+}\right)=X$.

3. When $\tau \rightarrow \infty$, we observe that $\widehat{c}(\tau ; r, q) \longrightarrow 0$ for $q>0$ and $r>0$ so that the exercise payoff for the perpetual single-reload option is the same as that of the perpetual American option. Since the governing equation and all auxiliary conditions of the two perpetual options are identical, the corresponding price functions are equal. It is well known that the price function of an American perpetual option is given by

$$
V_{A}^{\infty}(S)=\left(\frac{S_{A, \infty}^{*}}{\mu_{+}}\right)\left(\frac{S}{S_{A, \infty}^{*}}\right)^{\mu_{+}}
$$

where $S_{A, \infty}^{*}=\frac{\mu_{+}}{\mu_{+}-1} X$. It then follows that

$$
S_{1}^{*}(\infty ; r, q)=\frac{\mu_{+}}{\mu_{+}-1} X \quad \text { for } \quad q>0
$$


4. First, we consider the optimal exercise policy of the single-shout call option, whose price function is denoted by $c_{s h o u t, 1}(S, \tau ; X, r, q)$. A necessary condition for the point $(S, \tau)$ to lie in the stopping region of the single-shout call is given by (Dai et al., [4])

$$
\frac{d}{d \tau}\left[e^{-q \tau} \widehat{c}(\tau ; r, q)\right]=\widehat{c}^{\prime}(\tau ; \delta, 0)>0
$$

where $\delta=r-q$. Simple calculations show that

$$
\widehat{c}^{\prime}(\tau ; \delta, 0)=e^{-\delta \tau} f(\tau)
$$

where $f(\tau)=\delta N\left(d_{2}\right)+\frac{\sigma}{2 \sqrt{\tau}} n\left(d_{2}\right)$ and $d_{2}=\frac{\delta-\frac{\sigma^{2}}{2}}{\sigma} \sqrt{\tau}$. The sign behaviors of $\widehat{c}^{\prime}(\tau ; \delta, 0)$ and those of $f(\tau)$ are identical.

For $\delta \geq 0$, we have $f(\tau)>0$ for all $\tau>0$. However, when $\delta<0$, it can shown that $f(\tau)$ may be strictly positive for all $\tau>0$ or remains positive only for certain interval of $\tau$. Note that $f\left(0^{+}\right)=\infty$. We observe that $d_{2} \rightarrow-\infty$ as $\tau \rightarrow \infty$ and $\delta<0$, and consider

$$
\lim _{d_{2} \rightarrow-\infty} \frac{N\left(d_{2}\right)}{\frac{n\left(d_{2}\right)}{d_{2}}}=\lim _{d_{2} \rightarrow-\infty}-\frac{N\left(d_{2}\right)-d_{2} n\left(d_{2}\right)}{-d_{2} n\left(d_{2}\right)}=-1
$$

so that $f(\tau) \sim \frac{\delta+\frac{\sigma^{2}}{2}}{2} N\left(d_{2}\right)$ as $d_{2} \rightarrow-\infty$. We then have $f(\infty) \rightarrow 0^{-}$if $\delta<-\frac{\sigma^{2}}{2}$, and $f(\infty)>0$ if $-\frac{\sigma^{2}}{2} \leq \delta<0$. To examine the properties of the turning points of $f(\tau)$, we need to compute the derivative of $f(\tau)$ and obtain

$$
f^{\prime}(\tau)=\frac{\sigma^{2}}{4 \sqrt{\tau}}\left(\frac{\delta^{2}-\frac{\sigma^{4}}{4}}{\sigma^{2}}-\frac{1}{\tau}\right) n\left(d_{2}\right)
$$

The derivative $f^{\prime}(\tau)$ has the following properties:

(i) If $\delta<-\frac{\sigma^{2}}{2}$, then there is a unique solution to $f^{\prime}(\tau)=0$ over $(0, \infty)$.

(ii) If $0<\delta \leq-\frac{\sigma^{2}}{2}$, then $f^{\prime}(\tau) \leq 0$ over $(0, \infty)$.

Combining all the above results together, we can conclude that

(a) If $\delta=r-q \geq-\frac{\sigma^{2}}{2}$, then $f(\tau)>0$ for all $\tau>0$.

(b) If $\delta=r-q<-\frac{\sigma^{2}}{2}$, then there exists a unique value $\tau_{1}^{*} \in(0, \infty)$ such that $f(\tau)>0$ when $\tau<\tau_{1}^{*}$ and $f(\tau) \leq 0$ when $\tau \geq \tau_{1}^{*}$.

Referring back to the single-reload option, by virtue of Eq. (13), the roles of the dividend yield and interest rate in the price functions of the shout call and reload option are interchanged. With zero dividend yield and interest rate $r$ in the reload option, the corresponding value of $\delta$ in the shout call becomes $-r$. Hence we interpret the above results with respect to the single-reload option as follows:

(a) When $r \leq \frac{\sigma^{2}}{2}, S_{1}^{*}(\tau ; r, 0)$ is defined for all $\tau>0$. Also, we have $\lim _{q \rightarrow 0^{+}} \mu_{+}=1$ so that $\lim _{q \rightarrow 0^{+}} S_{1}^{*}(\infty ; r, q)=\infty$. 
(b) When $r>\frac{\sigma^{2}}{2}, S_{1}^{*}(\tau ; r, 0)$ is defined only for $\tau<\tau_{1}^{*} \in(0, \infty)$ where $\tau_{1}^{*}$ is the unique solution to

$$
f(\tau)=-r N\left(-\frac{r+\frac{\sigma^{2}}{2}}{\sigma} \sqrt{\tau}\right)+\frac{\sigma}{2 \sqrt{\tau}} n\left(-\frac{r+\frac{\sigma^{2}}{2}}{\sigma} \sqrt{\tau}\right)=0 .
$$

\section{APPENDIX B: Properties on $S_{n}^{*}(\infty ; r, q), q>0$}

Let $V_{n}^{\infty}(S ; X)$ denote the price function of the perpetual $n$-reload option, where $q>0$. The governing equation for $V_{n}^{\infty}(S ; X)$ is given by

$$
\frac{\sigma^{2}}{2} S^{2} \frac{\partial^{2} V_{n}^{\infty}}{\partial S^{2}}+(r-q) S \frac{\partial V_{n}^{\infty}}{\partial S}-r V_{n}^{\infty}=0, \quad 0<S<S_{n}^{*}(\infty) .
$$

The auxiliary conditions are

$$
\begin{aligned}
& V_{n}^{\infty}(0)=0, \\
& V_{n}^{\infty}\left(S_{n}^{*}(\infty)\right)=S_{n}^{*}(\infty)-X+X V_{n-1}^{\infty}(1 ; 1), \\
& \frac{d V_{n}^{\infty}}{d S}\left(S_{n}^{*}(\infty)\right)=1 .
\end{aligned}
$$

The solution to the above equation is given by

$$
V_{n}^{\infty}(S)=\left(\frac{S_{n}^{*}(\infty)}{\mu_{+}}\right)\left(\frac{S}{S_{n}^{*}(\infty)}\right)^{\mu_{+}}
$$

where

$$
S_{n}^{*}(\infty)=\frac{\mu_{+} X}{\mu_{+}-1}\left[1-V_{n-1}^{\infty}(1 ; 1)\right]
$$

Knowing that

$$
S_{1}^{*}(\infty)=\frac{\mu_{+} X}{\mu_{+}-1},
$$

we can deduce the recursive relation:

$$
\frac{S_{n}^{*}(\infty)}{X}=\frac{\mu_{+}}{\mu_{+}-1}-\frac{1}{\mu_{+}-1}\left[\frac{S_{n-1}^{*}(\infty)}{X}\right]^{1-\mu_{+}}, \quad n>1 .
$$

One can easily establish

$$
X<S_{n-1}^{*}(\infty)<S_{n}^{*}(\infty), \quad n>1,
$$

so that $\lim _{n \rightarrow \infty} S_{n}^{*}(\infty)$ exists. Let $L$ denote $\lim _{n \rightarrow \infty} S_{n}^{*}(\infty)$. From the above recursive relation, we deduce that $L$ satisfies

$$
\frac{L}{X}=\frac{\mu_{+}}{\mu_{+}-1}-\frac{1}{\mu_{+}-1}\left(\frac{L}{X}\right)^{1-\mu_{+}}, L \geq X .
$$

The unique solution to the above algebraic equation is $L=X$. 


\section{APPENDIX C: Proof of Eq. (3.8)}

Let $c_{\text {shout }, n}(S, \tau ; X, r, q)$ denote the price function of the $n$-shout call option. We take the limit $n \rightarrow \infty$ and define the price function of an infinite-shout call option by

$$
c_{\text {shout }}^{\infty}(S, \tau ; X, r, q)=\lim _{n \rightarrow \infty} c_{\text {shout }, n}(S, \tau ; X, r, q) .
$$

The governing equation for $c_{\text {shout }}^{\infty}(S, \tau)$ is given by

$$
\begin{aligned}
& \frac{\partial c_{\text {shout }}^{\infty}}{\partial \tau}-\mathcal{L}_{r, q} c_{\text {shout }}^{\infty}=0, \\
& S>S_{\text {shout }, \infty}^{*}(\tau), \quad \tau>0,
\end{aligned}
$$

with auxiliary conditions:

$$
\begin{aligned}
& c_{\text {shout }}^{\infty}\left(S_{\text {shout }, \infty}^{*}(\tau), \tau\right)=S_{\text {shout }, \infty}^{*}(\tau) c_{\text {shout }}^{\infty}(1, \tau ; 1), \\
& \frac{\partial c_{\text {shout }}^{\infty}}{\partial S}\left(S_{\text {shout }, \infty}^{*}(\tau), \tau\right)=c_{\text {shout }}^{\infty}(1, \tau ; 1), \\
& c_{\text {shout }}^{\infty}(S, 0)=(S-X)^{+} .
\end{aligned}
$$

The infinite-shout call has a simple exercise policy: the holder shouts when the option becomes in-the-money, that is, $S_{\text {shout }, \infty}^{*}(\tau)=X$ for all $\tau \geq 0$.

On the other hand, we consider the following pricing model of a floating strike lookback call option, whose price function is represented by $c_{f l o a t}(S, m, \tau ; r, q)$ :

$$
\frac{\partial c_{\text {float }}}{\partial \tau}-\mathcal{L}_{r, q} c_{\text {float }}=0, \quad S>m, \tau>0,
$$

with auxiliary conditions:

$$
\begin{aligned}
& \left.\frac{\partial c_{\text {float }}}{\partial m}\right|_{S=m}=0, \\
& c_{\text {float }}(S, m, 0)=S-m .
\end{aligned}
$$

We apply the following transformations

$$
\bar{c}_{\text {float }}(y, \tau)=\frac{c_{\text {float }}(S, m, \tau)}{m}, \quad y=\frac{S}{m},
$$

so that the governing equation of the pricing model of the lookback option can be transformed into

$$
\frac{\partial \bar{c}_{\text {float }}}{\partial \tau}-\mathcal{L}_{r, q} \bar{c}_{\text {float }}=0, \quad y>1, \tau>0,
$$

with auxiliary conditions:

$$
\begin{gathered}
\left.\frac{\partial \bar{c}_{\text {float }}}{\partial y}\right|_{y=1}=\bar{c}_{\text {float }}(1, \tau), \\
\bar{c}_{\text {float }}(y, 0)=y-1 .
\end{gathered}
$$

Comparing the pricing formulations of the infinite-shout call and the floating strike lookback call option, we then conclude that 
(a) when $S>X$,

$$
\begin{aligned}
& c_{\text {shout }}^{\infty}(S, \tau ; X) \\
= & X c_{\text {shout }}^{\infty}\left(\frac{S}{X}, \tau ; 1\right) \\
= & X \bar{c}_{\text {float }}\left(\frac{S}{X}, \tau\right)=c_{\text {float }}(S, X, \tau) .
\end{aligned}
$$

(b) when $S \leq X$,

$$
\begin{aligned}
& c_{\text {shout }}^{\infty}(S, \tau ; X) \\
= & S c_{\text {shout }}^{\infty}(1, \tau ; 1) \\
= & S \bar{c}_{\text {float }}(1, \tau)=c_{\text {float }}(S, S, \tau) .
\end{aligned}
$$

Combining the above results, we obtain

$$
c_{\text {shout }}^{\infty}(S, \tau ; X)=c_{\text {float }}(S, \min (X, S), \tau) .
$$

Next, we rewrite the pricing formulation of $W_{\infty}(x, \tau)$ into the following alternative form:

$$
\begin{aligned}
& \frac{\partial W_{\infty}}{\partial \tau}-\mathcal{L}_{q, r} W_{\infty}=q(x-1), \quad x>1, \tau>0 \\
& \frac{\partial W_{\infty}}{\partial x}-\left.W_{\infty}\right|_{x=1}=0 \\
& W_{\infty}(x, 0)=x-1 .
\end{aligned}
$$

Comparing the above formulation with the formulation of $\bar{c}_{\text {float }}(y, \tau)$, we then deduce that

$$
\begin{aligned}
& W_{\infty}(x, \tau) \\
= & c_{\text {float }}(S, \min (S, X), \tau ; q, r) \\
& +q \int_{0}^{\tau} c_{\text {float }}(S, \min (S, X), u ; q, r) d u .
\end{aligned}
$$

Lastly, the price function of the infinite-reload option is given by

$$
\begin{aligned}
& V_{\infty}(S, \tau ; X, r, q) \\
= & S W_{\infty}\left(\frac{X}{S}, \tau\right)+F(S, \tau) \\
= & c_{f l o a t}(S, \min (S, X), \tau ; q, r)+\left(S e^{-q \tau}-X e^{-r \tau}\right) \\
& +q \int_{0}^{\tau}\left[c_{\text {float }}(S, \min (S, X), u ; q, r)+\left(S e^{-q u}-X e^{-r u}\right)\right] d u .
\end{aligned}
$$

APPENDIX D: Proof of equivalence of the price formulas for infinite reload option 
Dybvig and Loewenstein [6] present the following price formula for an infinite-reload option:

$$
\begin{aligned}
& V_{\infty}(S, T ; X, r, q) \\
= & e^{-r T} E_{S_{T}}\left[S_{T}\left(1-\frac{X}{M_{T}}\right)^{+}\right] \\
& +q \int_{0}^{T} e^{-r u} E_{S_{u}}\left[S_{u}\left(1-\frac{X}{M_{u}}\right)^{+}\right] d u
\end{aligned}
$$

where the current time is taken to be the zeroth time and $M_{u}$ is the realized maximum value of the stock price over $(0, u)$. To prove the equivalence of their price formula with ours [see Eq. (3.8)], it suffices to show that

$$
\begin{aligned}
G_{0} & =e^{-r T} E_{S_{T}}\left[S_{T}\left(1-\frac{X}{M_{T}}\right)^{+}\right] \\
& =c_{\text {float }}\left(X, \min \left(X, S_{0}\right), T ; q, r\right)+S_{0} e^{-q T}-X e^{-r T} .
\end{aligned}
$$

We apply the transformations:

$$
H_{0}=\frac{G_{0}}{S_{0}}, x_{t}=\frac{X}{S_{t}} \quad \text { and } \quad m_{t}=\frac{X}{M_{t}}
$$

so that

$$
H_{0}=e^{-r T} E_{x_{T}}^{Q}\left[\left(1-m_{T}\right)^{+}\right] .
$$

We recognize $H_{0}$ as the value of a fixed strike lookback put option. By recalling the following parity relation between fixed strike lookback put and floating strike lookback call

$$
\begin{aligned}
& p_{\text {fix }}\left(x_{0}, m_{0}, T ; q, r\right) \\
= & c_{\text {float }}\left(x_{0}, \min \left(1, m_{0}\right), T ; q, r\right)-\left(x_{0} e^{-r T}-e^{-q T}\right),
\end{aligned}
$$

we deduce that

$$
H_{0}=c_{\text {float }}\left(x_{0}, \min \left(1, x_{0}\right), T ; q, r\right)-\left(x_{0} e^{-r T}-e^{-q T}\right) .
$$

Lastly, we obtain

$$
\begin{aligned}
G_{0} & =S_{0}\left[c_{\text {float }}\left(x_{0}, \min \left(1, x_{0}\right), T ; q, r\right)-\left(x_{0} e^{-r T}-e^{-q T}\right)\right] \\
& =c_{\text {float }}\left(X, \min \left(X, S_{0}\right), T ; q, r\right)+S_{0} e^{-q T}-X e^{-r T}
\end{aligned}
$$




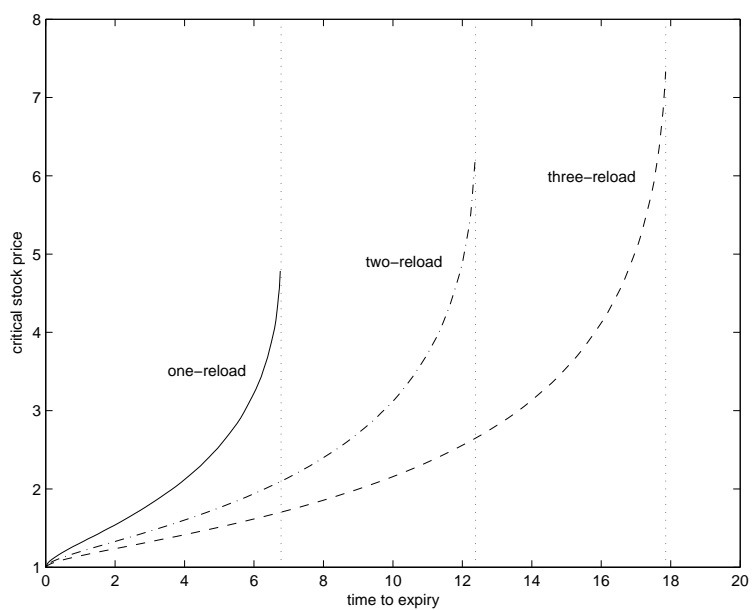

FIG. 1 Plots of critical stock price against time to expiry for employee options with one, two and three reloads $\left(q=0, r>\frac{\sigma^{2}}{2}\right)$. The parameter values used in the calculations are: $q=0, r=0.1, \sigma=0.3$ and $X=1$. The critical stock price $S_{n}^{*}(\tau ; r, q)$ is defined only for $\tau<\tau_{n}^{*}, n=1,2,3$. These threshold values on time to expiry are found to be: $\tau_{1}^{*}=6.78, \tau_{2}^{*}=12.38$ and $\tau_{3}^{*}=17.86$; and they observe the monotonic property: $\tau_{1}^{*}<\tau_{2}^{*}<\tau_{3}^{*}$.

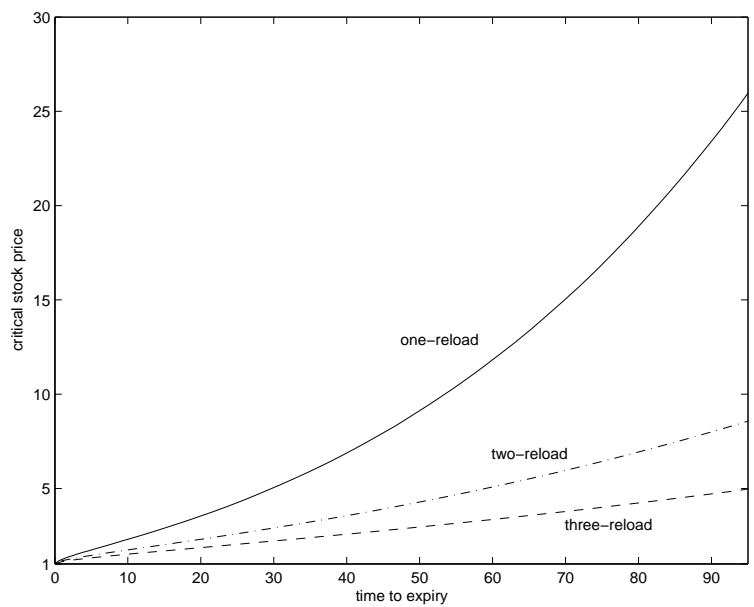

FIG 2. Plots of critical stock price against time to expiry for employee options with one, two and three reloads $\left(q=0, r \leq \frac{\sigma^{2}}{2}\right)$. The parameter values used in the calculations are: $q=0, r=0.04, \sigma=0.3$ and $X=1$. Apparently, the critical stock price is defined for all values of time to expiry and there is no asymptotic value for the critical stock price at infinite time to expiry. 


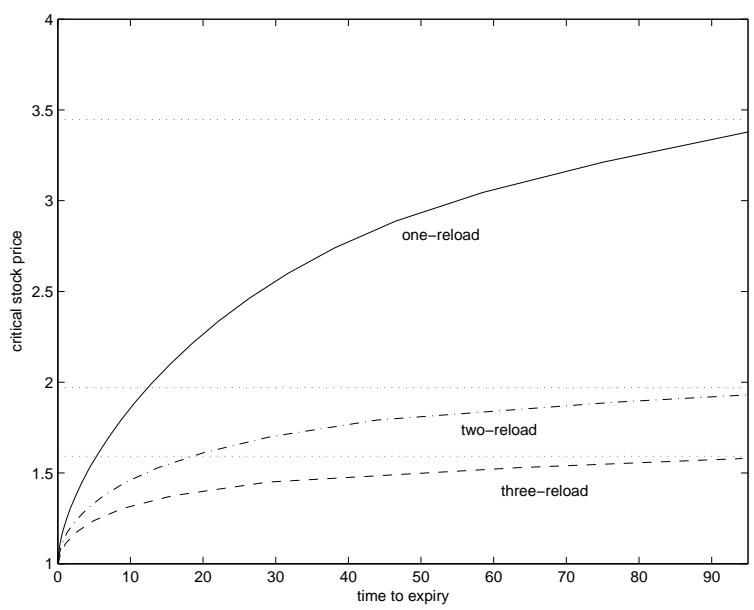

FIG 3. Plots of critical stock price against time to expiry for employee options with one, two and three reloads $(q>0)$. The parameter values used in the calculations are: $q=0.03, r=0.04, \sigma=0.3$ and $X=1$. The asymptotic values for the critical stock price at infinite time to expiry are found to be: $S_{1}^{*}(\infty)=3.45, S_{2}^{*}(\infty)=1.99$ and $S_{3}^{*}(\infty)=1.59$.

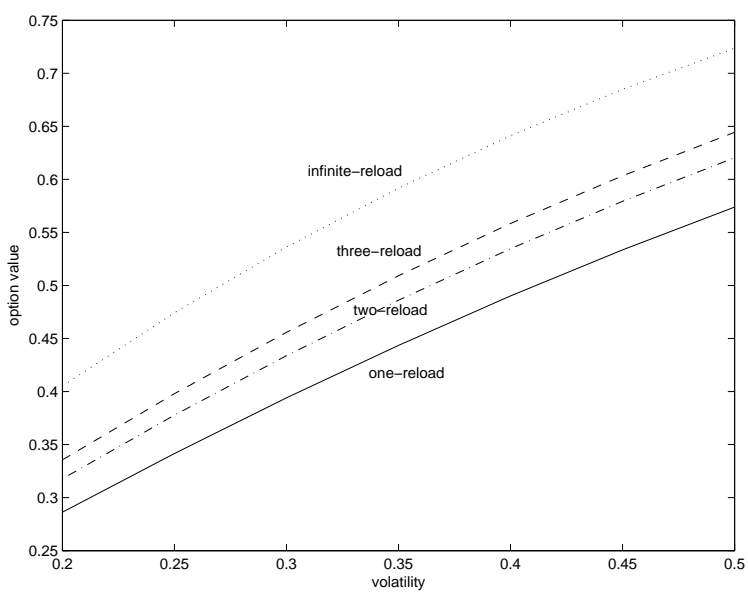

FIG 4. Plots of option value against interest rate for employee options with one, two, three and infinite reloads. The parameter values used in the calculations are: $q=0.03, r=0.04, \tau=1.0, S=X=1$. The price functions always increase in value with increasing volatility and increasing number of reloads outstanding. 